\title{
A small molecule inhibitor of dengue virus type 2 protease inhibits the replication of all four dengue virus serotypes in cell culture
}

Rajendra Raut ${ }^{1,2 \dagger}$, Hemalatha Beesetti ${ }^{3+}$, Poornima Tyagi $i^{1}$, Ira Khanna ${ }^{4}$, Swatantra K Jain ${ }^{2}$, Variam U Jeankumar ${ }^{5}$, Perumal Yogeeswari ${ }^{5,6}$, Dharmarajan Sriram ${ }^{5,6}$ and Sathyamangalam Swaminathan ${ }^{3,6^{*}}$

\begin{abstract}
Background: Dengue has emerged as the most significant of arboviral diseases in the 21st century. It is endemic to $>100$ tropical and sub-tropical countries around the world placing an estimated 3.6 billion people at risk. It is caused by four genetically similar but antigenically distinct, serotypes of dengue viruses. There is neither a vaccine to prevent nor a drug to treat dengue infections, at the present time. The major objective of this work was to explore the possibility of identifying a small molecule inhibitor of the dengue virus protease and assessing its ability to suppress viral replication in cultured cells.

Methods: We cloned, expressed and purified recombinant dengue virus type 2 protease. Using an optimized and validated fluorogenic peptide substrate cleavage assay to monitor the activity of this cloned dengue protease we randomly screened $\sim 1000$ small molecules from an 'in-house' library to identify potential dengue protease inhibitors.

Results: A benzimidazole derivative, named MB21, was found to be the most potent in inhibiting the cloned protease $\left(I C_{50}=5.95 \mu \mathrm{M}\right)$. In silico docking analysis indicated that MB21 binds to the protease in the vicinity of the active site. Analysis of kinetic parameters of the enzyme reaction suggested that MB21 presumably functions as a mixed type inhibitor. Significantly, this molecule identified as an inhibitor of dengue type 2 protease was also effective in inhibiting each one of the four serotypes of dengue viruses in infected cells in culture, based on analysis of viral antigen synthesis and infectious virus production. Interestingly, MB21 did not manifest any discernible cytotoxicity.

Conclusions: This work strengthens the notion that a single drug molecule can be effective against all four dengue virus serotypes. The molecule MB21 could be a potential candidate for 'hit-to-lead' optimization, and may pave the way towards developing a pan-dengue virus antiviral drug.
\end{abstract}

Keywords: Dengue fever, Dengue virus, NS2b-NS3 protease, Dengue protease inhibitor, Antiviral therapy

\section{Background}

Dengue is an arboviral disease which is currently a very significant global public health concern [1-3]. The disease is endemic to $>100$ tropical and sub-tropical countries. Of the $\sim 3.6$ billion people estimated to be at risk of dengue, 400 million people experience dengue infections annually [4]. Four antigenically distinct serotypes

\footnotetext{
* Correspondence: swaminathan@hyderabad.bits-pilani.ac.in

${ }^{\dagger}$ Equal contributors

${ }^{3}$ Department of Biological Sciences, Birla Institute of Technology and Science Pilani, Hyderabad Campus, Hyderabad 500078, India

${ }^{6}$ Centre for Infectious Disease Research, Birla Institute of Technology and

Science Pilani, Hyderabad Campus, Hyderabad 500078, India

Full list of author information is available at the end of the article
}

of dengue viruses (DENV-1, $-2,-3$ and -4 ) of the genus Flavivirus, family Flaviviridae, cause this disease [5]. Clinically, the disease has been distinguished as either mild dengue fever (DF) or potentially fatal dengue hemorrhagic fever (DHF) and dengue shock syndrome (DSS) [6]. Despite decades of efforts, a preventive dengue vaccine is not available $[7,8]$. Ongoing efforts have revealed the existence of challenging hurdles in dengue vaccine development [9-11]. This has spurred attention towards exploring the feasibility of developing therapeutic drugs $[12,13]$. Observations that the virus titers in DHF/DSS patients are an order of magnitude higher in comparison to DF patients $[14,15]$, suggest that a drug 
which can bring about $\sim 1 \log$ reduction in virus replication may be able to prevent the progression of DF to DHF/DSS.

The DENV genome is a single-stranded positive sense, 11 kilobases $(\mathrm{Kb})$ long RNA molecule [5]. It carries a 5' cap, but no poly A tail and contains a single large open reading frame (ORF) sandwiched between two non-translated regions located at either end. The ORF is translated in the infected host cell cytoplasm into $>3000$ amino acid (aa) residue long polyprotein precursor. Coand post-translational processing of this precursor by host and viral proteases generates ten viral proteins, of which three are structural and the rest, non-structural (NS) proteins [5]. One of these latter proteins, NS3, by virtue of its function as the viral protease is crucial in the polyprotein maturation process [16]. The protease activity of NS3, located in the $\mathrm{N}$-terminal one-third of the full-length molecule, contains the classic catalytic triad seen in serine proteases and relies on cofactor function, mapping to a hydrophilic 40 aa residue domain of another viral protein, NS2b [17]. Mutations in either component of the flaviviral protease that compromise its function lead to abrogation of replication $[18,19]$. This two component protease, NS2b-NS3Pro, has emerged as a potential antiviral drug target in recent years [16].

Ideally, a DENV inhibitor must be effective against all four DENV serotypes. This is because, each of the four DENVs can cause the full spectrum of dengue disease, and all four DENV serotypes tend to co-circulate in hyperendemic regions [2,3]. Functional profiling studies indicate that the NS2b-NS3Pro of the four DENV serotypes share very similar peptide substrate structure activity relationships [20]. Based on this notion, we have explored the feasibility of identifying an inhibitory molecule with pan-DENV-specificity by empirical screening of an 'in-house' library of $~ 1000$ small molecular weight compounds. This paper presents the identification of a DENV-2 NS2b-NS3 protease (NS2b-NS3Pro)-inhibitory molecule which could inhibit the replication of all four DENV serotypes in infected cells in culture.

\section{Results and discussion}

\section{Recombinant DENV-2 NS2b-NS3Pro}

We cloned and expressed DENV-2 protease in E. coli and purified it to $>90 \%$ homogeneity using modifications of previously reported methods [20-22]. The design of a synthetic NS2b-NS3Pro gene, its expression in E. coli and its purification by $\mathrm{Ni}^{2+}$-NTA affinity chromatography are summarized in Additional file 1: Figures S1 and S2. Using the synthetic fluorogenic peptide BenzoylNle-Lys-Arg-Arg-4-methylcoumarin-7-amide (Bz-nKRRAMC), which has been shown to be a better substrate compared to peptides containing endogenous dengue cleavage sites [20], we confirmed that our purified
DENV-2 NS2b-NS3Pro is enzymatically active based on the increase in fluorescence that accompanies peptide cleavage (Figure 1). Assay conditions were optimized to identify enzyme and substrate concentration ranges compatible with a linear dose-response (Figures 1A, and B). To validate this assay for inhibitor screening, we tested the effect of the protease inhibitor aprotinin, on the catalytic activity of DENV-2 NS2b-NS3Pro enzyme. Aprotinin is a serine protease inhibitor which can bind NS2b-NS3 strongly [20], and inhibit it effectively at nanomolar concentrations [21]. Our data showed that aprotinin inhibited the recombinant protease activity effectively $\left(\mathrm{IC}_{50}=20 \mathrm{nM}\right.$; Figure 1C).

\section{Compound MB21 inhibits DENV-2 NS2b-NS3Pro}

With a functionally validated DENV NS2b-NS3pro assay in hand, we next proceeded to screen an 'in-house' library of $\sim 1000$ small molecular weight compounds to identify potential inhibitors. Recent work has shown that this library contains antimicrobial compounds [23,24]. An initial screen wherein these compounds were tested at a single concentration $(25 \mu \mathrm{M})$, identified 25 compounds which manifested $>80 \%$ inhibition of the recombinant NS2b-NS3Pro. One of these, a benzimidazole compound, MB21, was the most potent, manifesting an $\mathrm{IC}_{50}$ of $5.9 \mu \mathrm{M}$ against the recombinant DENV-2 NS2bNS3pro enzyme (Figure 2A). Three additional benzimidazole compounds, RB02, RA14 and RA16, also inhibited the cloned DENV-2 protease, albeit at comparatively lower efficiency (Additional file 1: Figure S3). We used in silico molecular docking to understand how MB21 may interact with DENV-2 NS2b-NS3Pro. This analysis showed that MB21 bound to the DENV- protease with its benzimidazole moiety embedded well within the hydrophobic cleft of an allosteric site [25], in the vicinity of the catalytic triad, as depicted in Figure 2 (panels B and C). Features of MB21 binding observed here correlate with earlier reports on allosteric binding $[25,26]$. To understand better the mechanism of action of MB21 on DENV-2 NS2b-NS3Pro, we determined the efficiency of protease action over a range of substrate concentrations in the absence and presence of varying MB21 concentrations (Figure 3A). These data were analyzed using Lineweaver-Burke double reciprocal plot (Figure 3B). We observed that both the kinetic parameters, $K_{m}$ and $V_{\max }$, were changed by MB21. These data lead to the conclusion that MB21 may act by as a mixed inhibitor of DENV-2 NS2b-NS3Pro. This is consistent with the in silico docking data which show that MB21 binds to an allosteric site.

As a next step, we sought to assess if MB21 which inhibited cloned viral NS2b-NS3Pro efficiently had any inhibitory activity on the parent virus itself. To this end we used a cell-based assay in which we infected Vero 
A

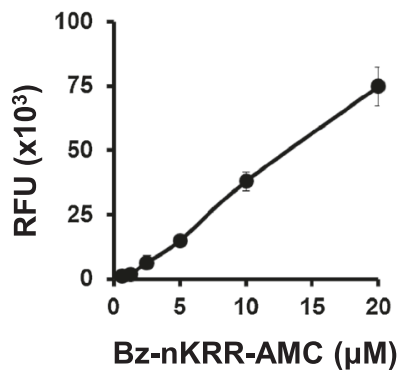

B

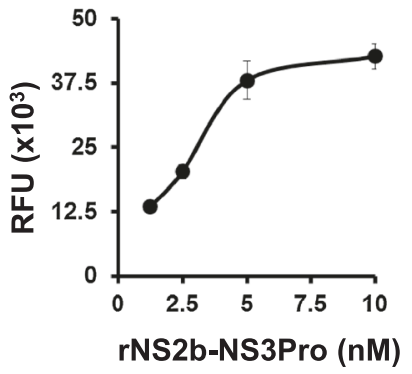

C

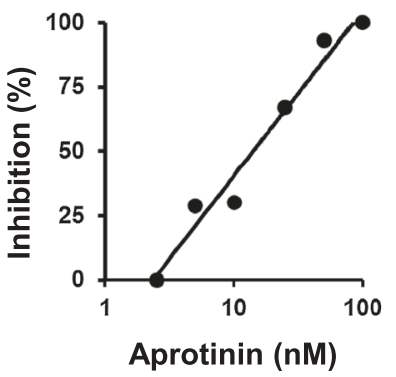

Figure 1 DENV-2 NS2b-NS3Pro enzyme assay, optimization and validation. (A) Kinetics of NS2b-NS3Pro action as a function of substrate concentration (at 5nM enzyme). (B) Rate of enzyme catalysis as a function of enzyme concentration (at $10 \mu \mathrm{M}$ substrate). (C) Activity of the cloned NS2b-NS3Pro as function of aprotinin concentration (5nM enzyme, $10 \mu \mathrm{M}$ substrate, 20 min incubation). Activity in the absence of aprotinin was taken as $100 \%$ (RFU = relative fluorescence units).

cells with DENV in the presence of MB21 and monitored the effect of the drug on virus replication. However, before testing MB21 for its DENV inhibitory potential, we sought to ascertain if this compound manifested any cytotoxicity on Vero cells. Interestingly, we found that MB21 at concentrations up to $50 \mu \mathrm{M}$ (in $0.5 \%$ DMSO vehicle) did not manifest any discernible cytotoxicity compared to controls (treated with $0.5 \%$ DMSO). This was essentially the case even at $100 \mu \mathrm{M}$ MB21 (in $1 \% \mathrm{DMSO}$ ), as evidenced by comparable cell viability between $100 \mu \mathrm{M}$ MB21-treated and 1\% DMSO-treated cells, as shown in Figure 4A. It is to be noted, however, that $1 \%$ DMSO by itself, caused $~ 30 \%$ loss of cell viability, compared to cells that were not treated with DMSO. Based on these results, we conclude that MB21 up to $100 \mu \mathrm{M}$ final concentration does not manifest discernible cytotoxicity on Vero cells.
It has been shown previously that DENV replication in cultured cells $[27,28]$ and in animal models [29] can be monitored by determining the levels of the viral antigen NS1 using immunoassays. Recently we showed that the levels of NS1 antigen secreted into culture supernatants of infected cells closely mirror the viral genomic RNA levels, measured using quantitative RT-PCR, for all four DENV serotypes [27].

\section{MB21 is a pan-DENV inhibitor}

Given the high degree of functional similarity of the NS2b-NS3Pro enzymes among the four DENV serotypes [20], we anticipated that MB21 may indeed be capable of inhibiting the replication of all four DENV serotypes. To test this, we infected Vero cells with each of the DENV serotypes separately, in the absence or presence of MB21 (at $30 \mu \mathrm{M}$ final concentration), and monitored

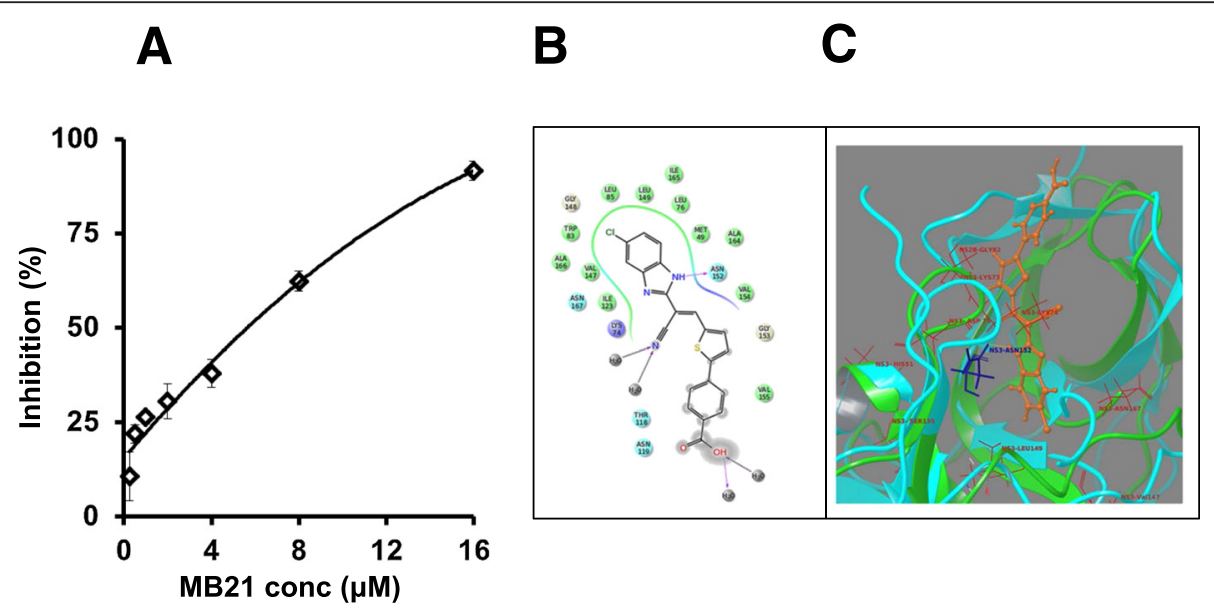

Figure 2 Inhibition of DENV-2 NS2b-NS3Pro by MB21 and in silico analysis of the interaction between the two. (A) Inhibition of protease activity of cloned DENV-2 NS2b-NS3Pro as a function of MB21 concentration. (B) Computer generated 2D ligand interaction picture depicting the interaction between MB21 and DENV-2 NS2b-NS3Pro. Hydrophobic residues are shown in green. (C) Interaction of MB21 at the allosteric pocket in the vicinity of the catalytic triad. 
A

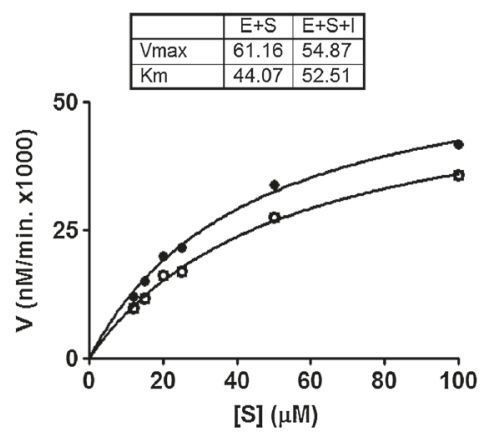

B

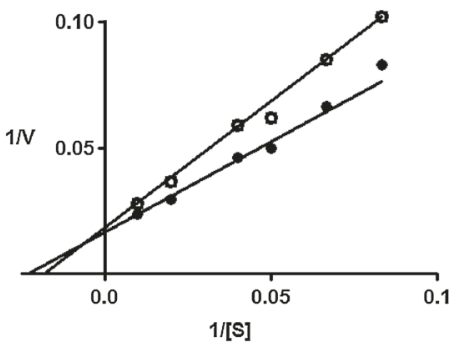

Figure 3 Mode of inhibition of DENV-2 NS2b-NS3Pro by MB21. (A) Enzymatic activity of DENV-2 NS2b-NS3Pro as a function of substrate concentration in the absence (filled circles) and presence of $6 \mu \mathrm{M}$ MB21 (empty circles). The table shows kinetic parameters in presence $(E+S+I)$ and absence $(E+S)$ of MB21. E, S and I denote the enzyme, DENV-2 NS2b-NS3Pro, the substrate, Bz-nKRR-AMC, and the inhibitor, MB21. (B) Lineweaver-Burke plot of DENV-2 NS2b-NS3Pro activity in the absence (filled circles) and presence of $6 \mu \mathrm{M} \mathrm{MB21} \mathrm{(empty} \mathrm{circles).} \mathrm{Data} \mathrm{were} \mathrm{analyzed} \mathrm{using}$ GraphPad Prism software.

NS1 antigen synthesis as a marker of DENV replication. Culture supernatants were withdrawn at regular intervals over a 1 week period and analyzed for NS1 antigen levels using a commercially available ELISA kit. The data comparing the kinetics of viral NS1 antigen secretion, by each one of the DENV serotypes, into the culture supernatant in the absence and presence of MB21 are presented in Figure 4 (panels $B$ to E). These results show that MB21 did indeed causes a statistically significant reduction of NS1 antigen levels secreted by all four DENV serotypes.

If NS1 secretion goes down, mirroring the downregulation of viral replication, it follows that final viral titers must also be reduced in the presence of MB21. To ascertain this possibility, we measured DENV titers in a virus yield reduction assay. In this experiment, viral titers in DENV-infected culture supernatants (MB21 treated as well as untreated) harvested at day 3 postinfection were measured using a standard plaque assay (Additional file 1: Figure S4). It was seen that at all dilutions of the culture supernatant tested, the number of plaques in the presence of MB21 was significantly lower compared to that in the absence of the drug. This experiment was performed with all four DENV serotypes. The viral titers calculated from the plaque counts are summarized in Table 1. These data reveal that MB21 could inhibit DENV-1, $-2,-3$ and -4 titers by $50,82,75$ and $73 \%$, respectively. This was found to be statistically significant. The observed reduction in DENV titers correlates with the decrease in viral antigen synthesis (Figure 4, panels B-E) and corroborates NS1 as a marker for DENV replication.

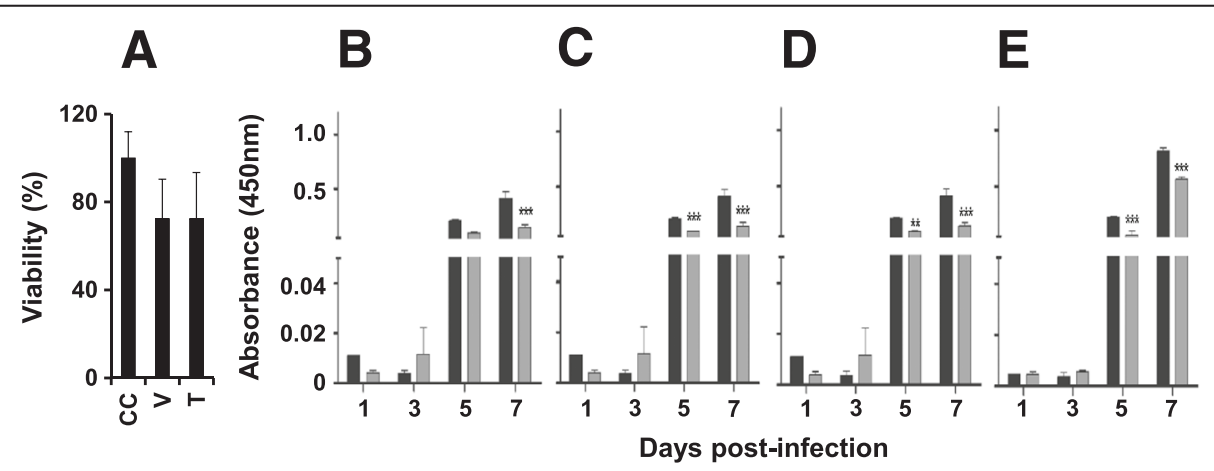

Figure 4 Evaluation of antiviral activity using cell-based assay. (A) Histogram showing the viability of Vero cells that received no drug ('CC', cell control), 1\% DMSO vehicle alone ('V') or $10 \mu \mathrm{M}$ MB21 in 1\% DMSO vehicle ('T'). Panels B-E depict the effect of MB21 on NS1 secretion by DENV-infected cells. Vero cells were infected with DENV-1 (B), DENV-2 (C), DENV-3 (D) or DENV-4 (E) either in the absence (black bars) or presence (grey bars) of MB21. Culture supernatants withdrawn at the indicated time points during the 1 week experiment, were tested for viral antigen levels using the Dengue NS1 ELISA kit (the NS1 ELISA absorbance scale on the Y-axis is the same for panels B-E). Data shown are mean values $(n=3)$. The vertical bars represent standard deviation, SD. Two-way ANOVA and Bonferroni post-test analysis was done using GraphPad Prism. $P$ values were either significant $\left(^{* *}\right)$ or very significant $\left(^{(* *)}\right.$. 


\begin{tabular}{|c|c|c|c|}
\hline DENV serotype $^{c}$ & Without MB21 & With MB21 ${ }^{d}$ & $P$ value ${ }^{e}$ \\
\hline 1 & $1.06 \pm 0.03$ & $0.52 \pm 0.02$ & $0.0028^{* * *}$ \\
\hline 2 & $1.54 \pm 0.12$ & $0.27 \pm 0.01$ & $0.0045^{* * *}$ \\
\hline 3 & $0.59 \pm 0.01$ & $0.14 \pm 0.04$ & $0.0065^{* * *}$ \\
\hline 4 & $0.22 \pm 0.02$ & $0.05 \pm 0.03$ & $0.031^{* *}$ \\
\hline
\end{tabular}

${ }^{a}$ Titers were determined by plaque assay on Vero cells.

${ }^{b} \mathrm{Pfu}=$ plaque forming units.

'The viral strains used were: DENV-1: West Pac 74; DENV-2: S-16803; DENV-3: CH54389; and DENV-4: TVP-360.

${ }^{d} \mathrm{MB} 21$ used at $30 \mu \mathrm{M}$ final concentration.

${ }^{e} P$ values were calculated using Graphpad software; $P$ values were either significant $\left.{ }^{* *}\right)$ or very significant $\left({ }^{* * *}\right)$.

Collectively, our data support the conclusion that MB21 is a pan-DENV inhibitor. The precise mechanism of the pan-DENV inhibitory activity of MB21 needs elucidation. Consistent with protease assay data, preliminary in silico docking (Figure 2) suggests that MB21 binds to the protease in the vicinity of its active site. The possibility that this binding may perturb the recently identified allosteric site (Ala125) on the protease [30] needs to be addressed. The likelihood that MB21 may compromise the ability of NS2b-NS3Pro to recruit fatty acid synthase during productive DENV infection [31] is another avenue to be explored.

\section{Conclusions}

The need for dengue drugs is being increasingly felt as dengue vaccine continues to be elusive. Based on its critical role in the DENV life cycle, NS2b-NS3Pro has emerged as a potential antiviral target. We set up and validated an in vitro DENV protease assay and used it to initiate a random screening campaign to search an inhouse' small molecule compound library, from which we have recently identified molecules with antimicrobial action $[23,24]$, for putative pan-DENV inhibitor(s). One molecule from this library, a benzimidazole compound, MB21, was a potent inhibitor of the cloned DENV-2 protease, NS2b-NS3Pro $\left(\mathrm{IC}_{50}=5.95 \mu \mathrm{M}\right)$. It appeared to bind to an allosteric site in the vicinity of the active site. Examination of steady state enzyme kinetics followed by double reciprocal plot analysis indicated that MB21 affects both $K_{m}$ and $V_{\max }$ and presumably functions as a mixed type inhibitor of NS2b-NS3Pro. Further it did not manifest significant cytotoxicity at concentrations as high as $100 \mu \mathrm{M}$. Interestingly, MB21 could suppress NS1 antigen secretion by all four DENVs, suggesting that it could function as a pan-DENV inhibitor. This was corroborated by plaque assay data which showed that viral titers were indeed reduced by MB21 in the case of each of the four DENV serotypes. The precise mechanism of action of MB21 on DENV replication needs to be elucidated. This molecule may provide a lead for further optimization.

\section{Methods}

\section{Cells, viruses, reagents}

The four DENV serotypes used in this study are the World Health Organization reference strains (DENV-1 West Pac 74, DENV-2 S-16803, DENV-3 CH54389 and DENV-4 TVP-360) and were kindly provided by Dr. De Silva, University of North Carolina, USA. The monkey kidney Vero cell line was from American Type Culture Collection, Virginia, USA. It was maintained in Dulbecco's Modified Eagle medium (DMEM), supplemented with $10 \%(\mathrm{v} / \mathrm{v})$ heat-inactivated $(\Delta)$ fetal calf serum (FCS), in a $10 \% \mathrm{CO}_{2}$ humidified incubator, at $37^{\circ} \mathrm{C}$.

The synthetic peptide substrate Bz-nKRR-MCA was custom-synthesized (Peptides International, Louisville, Kentucky, USA). NS1 ELISA kit was from J. Mitra \& Co. Pvt Ltd, New Delhi, India. MTT (3-(4, 5-dimethylthiazolyl-2)-2, 5 -diphenyltetrazolium bromide) assay kit was from Invitrogen (Life Technologies, USA).

The BITS in-house small molecule library consisted of diverse small molecules that included benzimidazoles, benzothiazoles, quinolones, thiazoles, thiazolidines, azetidines and spiropiperidones among others. Details of synthesis of the benzimidazoles identified as DENV-2 protease inhibitors are provided in Additional file 2.

\section{Protease and protease inhibition assays}

Protease assays were carried out in $100 \mu \mathrm{l}$ volume in microtiter wells of 96-well plates, essentially as described earlier [20]. A typical protease reaction $(100 \mu \mathrm{l})$ contained 5nM purified DENV-2 rNS2b-NS3Pro enzyme (15 ng protein) in assay buffer $(50 \mathrm{mM}$ Tris- $\mathrm{HCl}$, $\mathrm{pH} 8.5 / 1 \mathrm{mM}$ CHAPS/20\% glycerol). The reaction was initiated by the addition of peptide substrate Bz-nKRRAMC (10 mM stock) to a final concentration of $10 \mu \mathrm{M}$. The reaction was incubated at $37^{\circ} \mathrm{C}$ for 20 minutes. Protease activity was measured in terms of the increase in fluorescence that accompanied cleavage of the peptide substrate $\left(\lambda_{\mathrm{ex}}: 380 \mathrm{~nm} ; \lambda_{\mathrm{em}}: 450 \mathrm{~nm}\right)$. Control reactions in which the protease was omitted were run in parallel to correct for background fluorescence of the substrate. To measure protease inhibition, the test compound was incorporated into the protease reaction prior to substrate addition. Enzyme control (EC) reactions set up in parallel contained an equivalent amount of the vehicle (DMSO) without any inhibitor. Half maximal inhibitory concentration $\left(\mathrm{IC}_{50}\right)$ was defined as the inhibitor (test compound) concentration that decreased protease activity by $50 \%$, with reference to the EC reaction (which was taken as $100 \%)$, under the experimental conditions. All assays were run in duplicates or triplicates and each experiment was performed at least twice independently. 


\section{Vero cell-based DENV inhibition assay}

The titers of the stock viruses used were as follows: DENV-1: $1.6 \times 10^{6}$; DENV-2: $1.3 \times 10^{6}$; DENV-3: $1.5 \times 10^{7}$; \& DENV-4: $1.5 \times 10^{9} \mathrm{PFU} / \mathrm{ml}$. Vero cells were seeded in 48-well plates $\left(4 \times 10^{4}\right.$ cells/well in $0.5 \mathrm{ml} \mathrm{DME} \mathrm{+}$ $10 \% \triangle \mathrm{FCS})$ and incubated for 24 hours $\left(37^{\circ} \mathrm{C}, 5 \% \mathrm{CO}_{2}\right)$. Monolayers were aspirated and treated with $0.5 \mathrm{ml}$ $\mathrm{DME}+0.5 \%$ with $\triangle \mathrm{FCS}$ containing $30 \mu \mathrm{M}$ final concentration of the test compound. After 1 hour incubation medium + test compound was removed and saved. The monolayer was infected with DENV (m.o.i $=0.1 ; 200 \mu \mathrm{l}$ / well in DME $+0.5 \%$ with $\triangle \mathrm{FCS}$ ). After 2 hours, the virus inoculum was removed and replaced with medium containing test compound. The plate was returned to the incubator. Drug concentration was maintained by the addition of $5 \mu \mathrm{l}$ stock compound (equivalent to $30 \mu \mathrm{M}$ final concentration) solution into each well on days 3 and 5 post-infection. Aliquots $(20 \mu \mathrm{l})$ of culture supernatant were withdrawn at indicated time points up to 7 days for estimation of NS1 antigen by ELISA and viral titers by plaque assay. Appropriate virus controls (VC) for each DENV serotype, wherein the drug treatment was omitted, were run in parallel. All infection experiments were done twice independently. Inhibition by a test compound was assessed with reference to $\mathrm{VC}$ which was taken to represent $100 \%$ infectivity.

\section{DENV NS1 determination}

Culture supernatants collected at various time points, which were stored frozen at $-20^{\circ} \mathrm{C}$, were thawed and diluted appropriately (1:100 to $1: 1000$ in DME $+0.5 \% \triangle \mathrm{FCS}$ ). Suitable aliquots $(50 \mu \mathrm{l})$ of this were used to detect DENV NS1 antigen using a commercially available Dengue NS1 ELISA kit (J. Mitra \& Co., India), as per the manufacturer's protocol. This kit uses $\mathrm{N}$ - and C-terminal domain-specific anti-NS1 antibodies to detect the NS1 antigen produced by all four DENV serotypes.

\section{MTT assay}

Vero cells were seeded in a 96-well microtiter plate (5,000cells in $200 \mu \mathrm{l} \mathrm{DME}+5 \% \Delta \mathrm{FBS})$ were exposed to different test compounds at a range of concentrations $(2-100 \mu \mathrm{M})$ for four days at $37^{\circ} \mathrm{C}$ in a $10 \% \mathrm{CO}_{2}$ incubator. Control wells received an equivalent amount of DMSO vehicle without the test compound. Cell viability was assessed based on the reduction of MTT using a commercial kit as per the manufacturer's instructions.

\section{Statistical analysis}

The statistical significance between MB21-treated and untreated samples was assessed using GraphPad Prism v6 for Windows. Differences were considered statistically significant when the probability levels $(P)$ were $<0.05$.

\section{In silico studies}

Molecular docking of MB21 onto the three-dimensional crystal structure of DENV-2 NS2b-NS3Pro (pdb code: 2FOM) obtained from the protein data bank (www.rcsb.org) was performed using GLIDE extra precision module (Glide v5.7, Schrodinger, LLC, New York, NY) as described [32].

\section{Additional files}

Additional file 1: Supplementary results Figures S1-S4.

Additional file 2: Synthesis of benzimidazoles.

\section{Competing interests}

The authors declare that they have no competing interests.

\section{Authors' contributions}

RR carried out DENV infection assays, NS1 ELISAs and plaque assays. HB carried out the library screening work, participated in silico docking studies and helped with the initial manuscript draft. PT helped clone and purify the viral protease. IK and SKJ helped with study design. VUJ and DS carried out chemical synthesis. PY performed in silico docking studies. DS and PY helped in study coordination. SS conceived and designed the study, coordinated it and wrote the final manuscript. All authors read and approved the final manuscript.

\section{Acknowledgements}

The dengue type 2 protease clone was generated during the execution of a Department of Biotechnology, Government of India-funded project (SS) at ICGEB, New Delhi. SS acknowledges BITS Pilani University for providing start-up funds to initiate dengue research in the Hyderabad campus. HB is the recipient of an INSPIRE fellowship from the Department of Science \& Technology, Government of India. The authors are grateful to Prof. A. De Silva, University of North Carolina, USA, for the WHO reference panel of DENVs.

\section{Author details}

${ }^{1}$ Recombinant Gene Products Group, International Centre for Genetic Engineering and Biotechnology, New Delhi 110067, India. ${ }^{2}$ Department of Biotechnology, Jamia Hamdard, Hamdard Nagar, New Delhi 110062, India. ${ }^{3}$ Department of Biological Sciences, Birla Institute of Technology and Science Pilani, Hyderabad Campus, Hyderabad 500078, India. ${ }^{4}$ Department of General Medicine, Lady Hardinge Medical College, Shaheed Bhagat Singh Marg, New Delhi 110001, India. ${ }^{5}$ Department of Pharmacy, Birla Institute of Technology and Science Pilani, Hyderabad Campus, Hyderabad 500078, India. ${ }^{6}$ Centre for Infectious Disease Research, Birla Institute of Technology and Science Pilani, Hyderabad Campus, Hyderabad 500078, India.

Received: 18 November 2014 Accepted: 22 January 2015

Published online: 08 February 2015

\section{References}

1. Gubler DJ. The economic burden of dengue. Am J Trop Med Hyg. 2012;86:743-4

2. Guzman MG, Halstead SB, Artsob H, Buchy P, Farrar J, Gubler DJ, et al. Dengue: a continuing global threat. Nat Rev Microbiol 2010, Dec Supplement, S7-S16.

3. Swaminathan S, Khanna N. Dengue: recent advances in biology and current status of translational research. Curr Mol Med. 2009;9:152-73.

4. Bhatt S, Gething PW, Brady OJ, Messina JP, Farlow AW, Moyes CL, et al. The global distribution and burden of dengue. Nature. 2013:496:504-7.

5. Lindenbach BD, Thiel HJ, Rice CM. Flaviviridae: the viruses and their replication. In: Knipe DM, Howley PM, editors. Field's Virology. 5th ed. Philadelphia: Wolters Kluwer and Lippincott Williams \& Wilkins; 2007. p. 1101-52.

6. World Health Organization: Factsheet $N^{\circ} 117$ : Dengue and dengue haemorrhagic fever. 2014. [www.who.int/mediacentre/factsheets/fs117/en/]

7. Swaminathan S, Batra G, Khanna N. Dengue vaccines: state of the art. Exp Opin Ther Patents. 2010;20:819-35. 
8. Swaminathan S, Khanna N. Experimental dengue vaccines. In: Giese M, editor. Molecular Vaccine-from prophylaxis to therapy. Heidelberg: SpringerVerlag; 2013. p. 135-51.

9. Capeding MR, Tran NH, Hadinegoro SRS, Ismail HIHJM, Chotpitayasunondh $T$, Chua MN, et al. Clinical efficacy and safety of a novel tetravalent dengue vaccine in healthy children in Asia: a phase 3, randomized, observermasked, placebo-controlled trial. Lancet. 2014;384:1358-65.

10. Sabchareon A, Wallace D, Sirivichayakul C, Limkittikul K, Chanthavanich P, Suvannadabba $S$, et al. Protective efficacy of the recombinant, live-attenuated, CYD tetravalent dengue vaccine in Thai schoolchildren: a randomized, controlled phase 2b trial. Lancet. 2012;380:1559-67.

11. Swaminathan S, Khanna N, Herring B, Mahalingam S. Dengue vaccine efficacy trial: does interference cause failure? Lancet Infect Dis. 2013;13:191-2.

12. Lim SP, Wang QY, Noble CG, Chen YL, Dong H, Zou B, et al. Ten years of dengue drug discovery: progress and prospects. Antiviral Res. 2013;100:500-19.

13. Noble CG, Chen YL, Dong H, Gu F, Lim SP, Schul W, et al. Strategies for development of dengue virus inhibitors. Antiviral Res. 2010;85:450-62.

14. Libraty DH, Endy TP, Houng HSH, Greene S, Kalyanarooj S, Suntayakorn S, et al. Differing influences of virus burden and immune activation on disease severity in secondary dengue-3 virus infections. J Infect Dis. 2002;185:1213-21.

15. Vaughn DW, Greene S, Kalayanarooj S, Innis BL, Nimmanitya S, Suntayakorn $S$, et al. Dengue viremia titer, antibody response pattern, and virus serotype correlate with disease severity. J Infect Dis. 2000;181:2-9.

16. Lescar J, Luo D, Xu T, Sampath A, Lim SP, Canard B, et al. Towards the design of antiviral inhibitors against flaviviruses: the case for the multifunctional NS3 protein from dengue virus as a target. Antiviral Res. 2008;80:94-101.

17. Falgout $\mathrm{B}$, Miller RH, Lai CJ. Deletion analysis of dengue virus type 4 nonstructural protein NS2b: identification of a domain required for NS2b-NS3 protease activity. J Virol. 1993;67:2034-42.

18. Chambers TJ, Weir RC, Grakoui A, McCourt DW, Bazan JF, Fletterick RJ, et al. Evidence that the $\mathrm{N}$-terminal domain of nonstructural protein NS3 from yellow fever virus is a serine protease responsible for site-specific cleavage in the viral polyprotein. Proc Natl Acad Sci USA. 1990;87:8898-902.

19. Chambers TJ, Nestorowicz A, Amberg SM, Rice CM. Mutagenesis of the yellow fever virus NS2b protein: effects on proteolytic processing, NS2b-NS3 complex formation, and viral replication. J Virol. 1993;67:6797-807.

20. Li J, Lim SP, Beer D, Patel V, Wen D, Tumanut C, et al. Functional profiling of recombinant NS3 proteases from all four serotypes of dengue virus using tetrapeptide and octapeptide substrate libraries. J Biol Chem. 2005;280:28766-74.

21. Leung D, Schroder K, White H, Fang NX, Stoermer MJ, Abbenante G, et al. Activity of recombinant dengue 2 virus NS3 protease in the presence of a truncated NS2b co-factor, small peptide substrates, and inhibitors. J Biol Chem. 2001;276:45762-71.

22. Yusof R, Clum S, Wetzel M, Murthy HMK, Padmanabhan R. Purified NS2B/ NS3 serine protease of dengue virus type 2 exhibits cofactor NS2B dependence for cleavage of substrates with dibasic amino acids in vitro. J Biol Chem. 2000;275:9963-9.

23. Jean Kumar VU, Poyraz O, Saxena S, Schnell R, Yogeeswari P, Schneider G, et al. Discovery of novel inhibitors targeting the Mycobacterium tuberculosis O-acetylserine sulfhydrylase (CysK1) using virtual high-throughput screening. Biorg Med Chem Lett. 2013;23:1182-6.

24. Poyraz O, Jeankumar VU, Saxena S, Schnell R, Haraldsson M, Yogeeswari $P$, et al. Structure-guided design of novel thiazolidine inhibitors of O-acetyl serine sulfhydrylase from Mycobacterium tuberculosis. J Med Chem. 2013;56:6457-66.

25. Mukhametov A, Newhouse El, Aziz NA, Saito JA, Alam M. Allosteric pocket of the dengue virus (serotype 2) NS2B/NS3 protease: In silico ligand screening and molecular dynamics studies of inhibition. J Mol Graph Model. 2014;52:103-13.

26. Othman R, Kiat TS, Khalid N, Yusof R, Newhouse El, Newhouse JS, et al. Docking of noncompetitive inhibitors into dengue virus type 2 protease: understanding the interactions with allosteric binding sites. J Chem Inf Model. 2008;48:1582-91.

27. Korrapati AB, Swaminathan G, Singh A, Khanna N, Swaminathan S. Adenovirus-delivered short hairpin RNA targeting a conserved site in the $5^{\prime}$ non-translated region inhibits all four serotypes of dengue viruses. PLoS Negl Trop Dis. 2012;6:e1735.
28. Ludert JE, Mosso C, Ceballos-Olvera I, del Angel RM. Use of a commercial enzyme immunoassay to monitor dengue virus replication in cultured cells. Virol J. 2008;5:51.

29. Schul W, Liu W, Xu HY, Flamand M, Vasudevan SG. A dengue fever viremia model in mice shows reduction in viral replication and suppression of the inflammatory response after treatment with antiviral drugs. J Infect Dis. 2007;195:665-74.

30. Yildiz M, Ghosh S, Bell JA, Sherman W, Hardy JA. Allosteric inhibition of the NS2b-NS3 protease from dengue virus. ACS Chem Biol. 2013;8:2744-52.

31. Heaton NS, Perera R, Berger KL, Khadka S, LaCount DJ, Kuhn RJ, et al. Dengue virus non-structural protein 3 redistributes fatty acid synthase to sites of viral replication and increases cellular fatty acid synthesis. Proc Natl Acad Sci USA. 2010;107:17345-50.

32. Friesner RA, Banks JL, Murphy RB, Halgren TA, Klicic JJ, Mainz DT, et al. Glide: a new approach for rapid, accurate docking and scoring. 1. Method and assessment of docking accuracy. J Med Chem. 2004;47:1739-49.

\section{Submit your next manuscript to BioMed Central and take full advantage of:}

- Convenient online submission

- Thorough peer review

- No space constraints or color figure charges

- Immediate publication on acceptance

- Inclusion in PubMed, CAS, Scopus and Google Scholar

- Research which is freely available for redistribution 